\title{
The research of destruction of functionally- graded materials subjected to impact loading
}

\author{
Maxim Yu. Orlov ${ }^{1}$, Viktor Glazyrin ${ }^{1}$ and Yulia Orlova $^{2}$ \\ ${ }^{1}$ Tomsk State University, Scientific Research Institute Applied of Mathematics and Mechanics, 36, \\ Lenin Avenue, Tomsk, 634050, Russia \\ ${ }^{2}$ Tomsk Polytechnic University, Division for Oil and Gas Engineering, 30, Lenin Avenue, Tomsk, \\ 634050, Russia
}

\begin{abstract}
A numerical analysis on impact response of multilayer plates and plates with a gradient substrate against steel projectile perforation was made. The shear strength was varied in the substrate within a certain range. The behavior of bodies is modeled by an elastic-plastic, porous, compressible medium, taking into account shock-wave phenomena and fragmentary fracture of materials. A numerical lagrangian method with modified node splitting algorithms was used. Good agreement between the computed and experimental results was obtained. During perforation, pattern of destruction of all plates has been investigated. The results show impact resistance of plates with a gradient substrate was greater than the homogeneous steel one, but less than multilayer ones. However, the impact resistance of multilayer plates is explained by the pinching effect of the layers.
\end{abstract}

\section{Introduction}

Currently, increasing the shock resistance of protective structures is relevant and complex scientific and technical problem. Well-known fact is that the existing nomenclature of metals does not meet those requirements. In addition to, armour-piercing projectiles constitute a major issue when designing for personal protective and vehicle protection [1].

This fact makes researcher team to seek new ways to improve the impact resistance of protective structures. Numerical modeling is the most informative and convenient research tool. Recently to use a gradient of strength properties of materials against impact loading was proposed and this was discussed in [2]. It is presence gradient unidirectional substrate in metal specimen that is slightly increased impact resistance against normal impact below ballistic limit. The impact resistance of a bi-directional gradient specimen is significantly higher than that of a homogeneous steel one under the same conditions. The current study investigated the ballistic performance of single-layer, multi-layer and steel targets with a gradient substrate impacted by ogive-nosed projectile.

The present paper will mainly describe and discuss the ballistics performance targets impacted $6.1-\mathrm{mm}$-projectile at $500 \mathrm{~m} / \mathrm{s}$ by using numerical method, which combined with experimental results. The paper is structured as follows: phenomenological model of material behavior and numerical method, including test calculation are stated in section 2; 
the description all targets, including gradient one and numerical simulation they perforation with projectile presented in section 3; Section 4 analyzed ballistics performance all target.

\section{Model and method}

This section presents a phenomenological model of the behavior of bodies and a numerical method based on the lagrangian approach to describing the motion of continuous media. A numerical method for a 2D statement is also presented. The model and the method were developed at the and thoroughly tested. According to [3] we have in mind the conduct of internal, qualitative and quantitative tests. The mathematical model is based on the macroscopic theory of continuum mechanics. The numerical method is the development of the G.R. Johnson's one for penetration and perforation problems [4]. According to [5], the numerical method contains a new way for isolating discontinuity surfaces of materials, which does not impose serious restrictions on the solution of the problems.

\subsection{A mathematical model}

The mathematical model makes it possible to adequately describe the deformation and destruction of materials in a wide range of initial conditions. The system of governing equations is based on the fundamental laws of conservation of mass, momentum, and energy. To describe the shear strength of a body, the Prandtl - Reuss constitutive equations and the von Mises yield condition were used. The equation of state was Mi - Grüneisen. A complex continuum mechanics model with a deterministic approach to describing material failure was applied. Material was modeled as elastic-plastic, porous, compressible medium taking into account the strength properties, shock-wave phenomena and material failure. This model can describe the destruction of metallic and ceramic materials also.

\subsection{A numerical method and a test calculations}

In this subsection, we focus on the numerical method. The system of equations is solved in the two-dimensional axisymmetric statement on the basis of the Lagrangian approach to the description of the motion of continuous media. Well-known fact is that any lagrangian method has serious ones with modelling penetration and perforation problems. For instance, ones are the penetrating of multi-layer targets by an ogive-nosed projectile. The algorithm of erosion elements, algorithm of splitting nodes, and algorithm for constructing the free surface were implemented to overcome the problem of the elements overlapping under large deformation. A similar approach to modeling the perforation problems developed in the work [6]. However, in the present approach, several ways of splitting nodes are possible.

By the test problem, we mean the problem of deep penetration of the projectile into a semi-infinite target. The projectile velocity is $536 \mathrm{~m} / \mathrm{s}$, the material of the plate is $\mathrm{AMg} 6 . \mathrm{In}$ accordance to [3, we conducted a quantitative test, the subject of comparison of which was the penetration depth of the ogive-nosed projectile. Good agreement between the computed and experimental data was noted [7]. The experimental penetration depth was $15.3 \mathrm{~mm}$, and the computed penetration depth was $15.6 \mathrm{~mm}$.

\section{Numerical simulation results}


This section focuses on the results of numerical simulation of the perforation of all targets, including plate with gradient substrate. The graphs of the time dependences of the residual projectile velocity and the damage of the material were built. We do not show material damage curves because they were of the same type. The damage parameter is taken [7]. The configurations "Projectile - target" are analyzed in detail at all stages of perforation.

\subsection{A projectile and a target}

We used one projectile and six metal targets. The projectile is a core of the German SMK bullet (diameter is $6.1 \mathrm{~mm}$, height is $16 \mathrm{~mm}$, weight is $2.55 \mathrm{~g}$ ). In what follows, we will call it the ogive-nosed projectile. The projectile in numerical simulations consists of highstrength alloy U10A steel. Projectile is the same as in test calculation (See 2.2).

The first two targets are homogeneous steel plates 4 and $6 \mathrm{~mm}$ thick, respectively. The third target is a two-layer plate, the thickness of the upper layer is $4 \mathrm{~mm}$, the thickness of the lower layer is $2 \mathrm{~mm}$. The fourth target has an air gap. The thickness of the air gap is equal to the thickness of the bottom layer, i.e. $1 \mathrm{~mm}$. The fifth target contained 3 layers. The top layer is $4 \mathrm{~mm}$, and the bottom ones are $1 \mathrm{~mm}$ each. The last target has a $2-\mathrm{mm}$ gradient substrate. Target material is low steel alloys. Target's designation are (4), (6), $(4+2),(4+0+1),(4+1+1)$ and $\left[6^{\uparrow}\right]$, respectively.

\subsubsection{A target with gradient substrate}

In this subsection, we will talk about the object of research, which is a steel plate with a gradient substrate. The gradient substrate was located near at the back of the plate. The thickness of the substrate was $2 \mathrm{~mm}$, that is, equal to the thickness of the lower layer in the target (4+2). The "adhesion condition" was set between the gradient and homogeneous parts of the target. In the substrate, the shear strength linearly increased in the range from 50 to $55 \mathrm{~kJ} / \mathrm{kg}$. It is obvious that the range of its variation corresponds to real steel alloys. The shear strength in homogeneous steel is $45 \mathrm{~kJ} / \mathrm{kg}$, i.e. less than in substrate one. The rest of the strength characteristics, including the shear modulus, yield strength, spall strength in the steel plate and the gradient substrate, were the same. The recipe composition of the substrate was selected based [2].

\section{Results and discussion}

A set of computational experiments included 6 cases, initial velocity was $500 \mathrm{~m} / \mathrm{s}$. Fig. 1 shows the results of numerical simulations for all six cases. Since the results are axisymmetric, the left part of the figure corresponded to one case, and the right part of the figure corresponded to another. According to the computed data all the targets were perforated with projectile. It was found that the first foci of destruction are formed in the contact zone of the projectile and the target. The destruction zone was not extended to the lateral side surface of the target. The diameter of the holes in the targets was almost equal to the diameter of the projectile. Projectile's nose was subjected to minimal erosion of the tip. The projectile pierced plates. Small fragments of the perforated plate formed ahead of the projectile. After perforating targets $(4+2)$ and $(4+1+1)$ an air gap was formed between the layers. All the lower layers had a noticeable plastic deformation in the axial direction. After $70 \mu \mathrm{s}$, the projectile reached a constant velocity in all computational cases. 

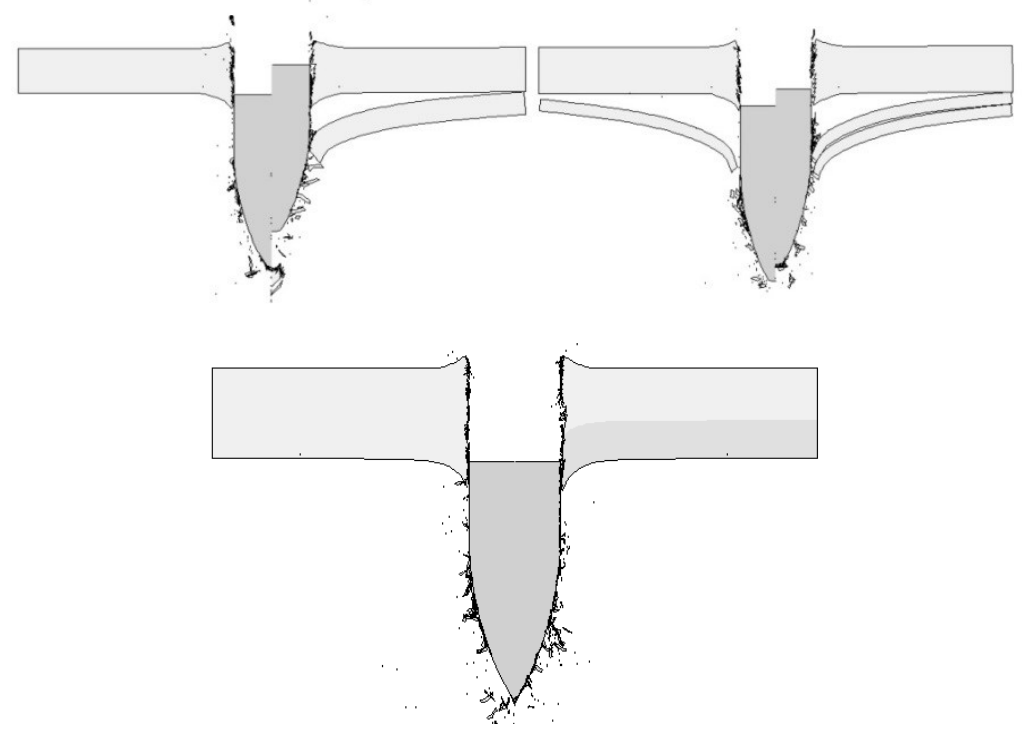

Fig.1. Numerical models of a projectile and all targets: across sectional target (4) and (4+2) at $42 \mu \mathrm{s}$ (see top left picture), cross sectional target $(4+0+1)$ and $(4+1+1)$ at $60 \mu$ s (see top right picture), cross sectional target (6) and $\left[6^{\uparrow}\right]$ at $60 \mu$ s (see bottom picture).

Results for all steel plates (see Table 1) are shown in Fig. 2 for comparison. It can be seen that the triple-layered steel plate $(4+1+1)$ and gradient plate $\left[6^{\uparrow}\right]$ has the best ballistic performance while homogeneous plate (4) has the poorest performance. The target $(2+4)$ had closest performance to target $\left[6^{\uparrow}\right]$.

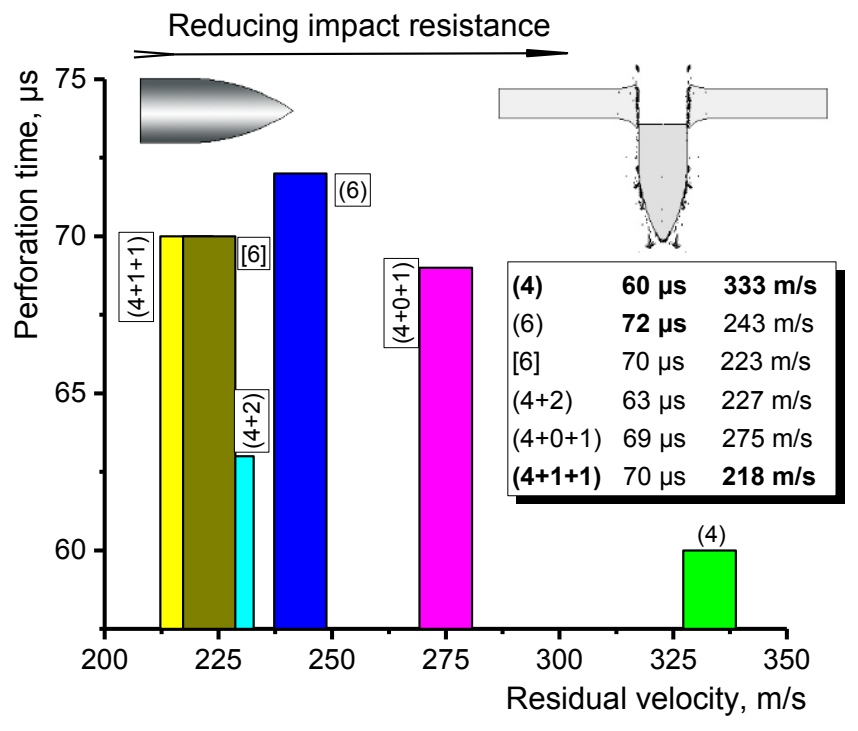

Fig.2. Perforation time Versus Residual velocity 


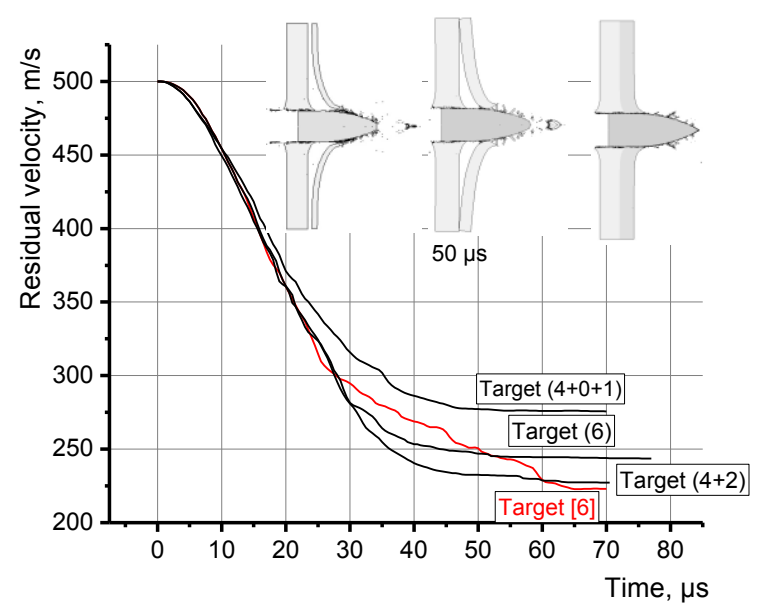

Fig.3. Perforation of double layered plates $(4+0+1)$ and $(4+2)$, homogeneous plate $(6)$ and plate with gradient substrate $\left[6^{\uparrow}\right]$ for initial impact velocity $500 \mathrm{~m} / \mathrm{s}$

Fig. 3 shows projectile velocity versus time for $(6),\left[6^{\uparrow}\right],(2+4),(4+0+1)$ plates when impacted with an initial velocity of $500 \mathrm{~m} / \mathrm{s}$. Perforation of plates at $50 \mu \mathrm{s}$ was also depicted. It can be seen that the mechanisms involved in the penetration process was different for each case and they define the difference in the ballistic performance. The velocity curves had sections of rapid decrease in velocity and sections of its slow decrease. Moreover, the sections of rapid decrease in velocity were at the beginning of the perforation process. The velocity curve for the gradient plate $\left[6^{\uparrow}\right]$ differed from the velocity curve for the homogeneous plate (6).

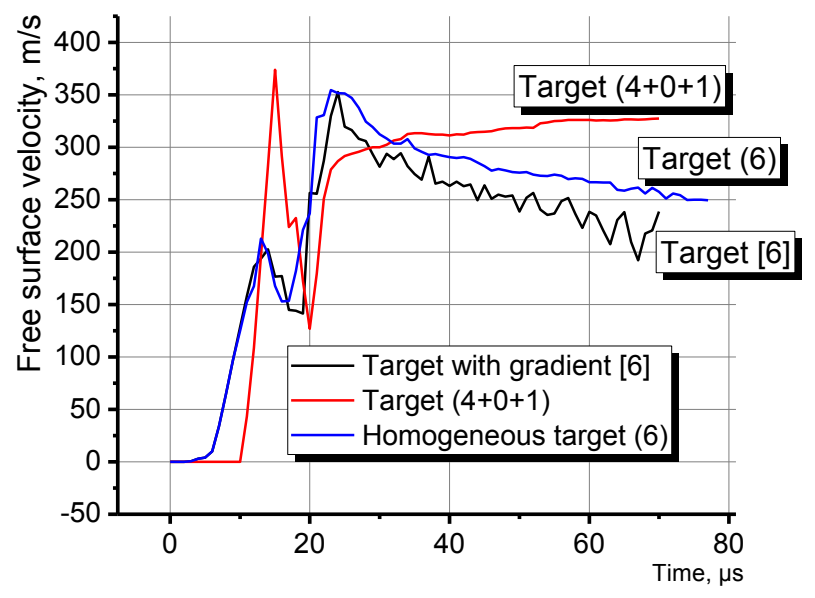

Fig.4. Free surface velocity versus Time

Fig. 4 shows the free surface velocity for some target. Previously, we simulated the free surface velocity from [8] and compared the experimental and calculated profiles. Good agreement was obtained.

The velocity profiles had different shapes. However, the shapes are typical for a wide class of metals. The maximum drop in velocity after the exit of the shock wave on the free 
surface was noted for the target $(4+0+1)$. Oscillations of the velocity are marked on the curve for $\left[6^{\uparrow}\right]$. The velocity of the free surface of the gradient plate was less than the velocity of a homogeneous plate.

Six different targets were subjected to a high-velocity impact to evaluate their impact resistance as summarized in Table 1.

Table 1. Numerical results

\begin{tabular}{l|c|c|c|c|c|c}
\hline & $\mathbf{( 4 )}$ & $\mathbf{( 6 )}$ & $\mathbf{( 4 + 2 )}$ & $\mathbf{( 4 + 0 + 1 )}$ & $\mathbf{( 4 + 1 + 1 )}$ & {$\left[\mathbf{6}^{\uparrow}\right]$} \\
\hline Residual velocity, $\mathrm{m} / \mathrm{s}$ & 333 & 243 & 227 & 275 & 218 & 223 \\
\hline Perforation time, $\mu \mathrm{s}$ & 60 & 72 & 63 & 69 & 70 & 73 \\
\hline Damage material, \% & 6,03 & 5,85 & 5,38 & 5,88 & 5,56 & 5,76 \\
\hline Hole diameter, $\mathrm{cm}$ & 0,614 & 0,666 & 0,69 & 0,62 & 0,74 & 0,75 \\
\hline
\end{tabular}

The maximum residual projectile velocity was after perforation the plate (4), and the minimum was after perforation plate $(4+1+1)$. In last case we explained it by the pinching effect of the layers $[7,9]$. The difference velocity between the gradient plate $\left[6^{\uparrow}\right]$ and the three-layer plate is negligible. But the difference between a homogeneous plate (6) and a gradient plate of the same thickness was almost $20 \mathrm{~m} / \mathrm{s}$.

It can be seen that the perforation time for all plates does not exceed $72 \mu \mathrm{s}$. By the time of perforation, we mean the time the back surface of the projectile reaches the back of the plate (see Fig 2). The minimum time was for plate (4). Maximum time was for plates (6) and $\left[6^{\uparrow}\right]$. The perforation time of plates $(4)$ and $(4+2)$ differed by only $5 \%$. The perforation process proceeded according to approximately similar mechanism.

The damage to the material and the diameter of hole in plate also differed quite a bit. So the damage in the material of plates was no more than $6 \%$. The damage accumulation time in the plates did not exceed $2 / 3$ of perforation process. The diameter of the holes in the plates almost coincided with the original diameter of the projectile.

The presence of a graded substrate in a steel sample only slightly increases its impact resistance against an ogive-nosed projectile. A slight decrease in the amount of material damage and an increase in perforation time were noted.

This study was supported by the RFBR (Project No. 19-08-01152a).

\section{References}

1. Y. Duplan, D. Saletti, P. Forquin, Identification of Johnson-Cook model parameters of an AP projectile core based on two shear-compression specimen geometries and one dog-bone sample, in Proceedings of the Light Weight Armour Group for Defense and Security (Roubaix, France) (2019)

2. M.Yu. Orlov, Yu. N. Orlov, V.P. Glazyrin and Yu. N Orlova, EPJ Web of Conferences 183, 01049 (2018)

3. V. Selivanov, Means of destruction and ammunition (BMTU Publishing, Moscow, 2008)

4. T. Holmquist and G. Johnson JAM 78 051003-1 (2011)

5. V.M. Fomin, Interaction of solids (SB RAS Publishing, Novosibirsk, 1999)

6. L. Olovsson, J. Limido, J. Lacome, A.G. Hanssen, J. Petit EPJ, 94, 04050 (2015)

7. A. Gerasimov, Studies of high-speed interaction of bodies (TSU Publishing, Tomsk, 2007)

8. S.J. Fensin, E.K. Walker, E.K. Cerreta, G.T. Gray III EPJ, 94, 02010 (2015)

9. G. Ben Dor, A. Dubinsky T. Evperin Engineering models in high-speed penetration mechanics and their applications (WSP Publishing, Singapore, 2019) 